\title{
On Redundant Index Assignments for Iterative Source-Channel Decoding
}

\author{
Thorsten Clevorn, Laurent Schmalen, Peter Vary, and Marc Adrat
}

\begin{abstract}
Iterative source-channel decoding (ISCD) exploits the residual redundancy of source codec parameters by using the Turbo principle. A powerful improvement for ISCD is the concept of redundant index assignments. In this letter we present new design and optimization guidelines for redundant index assignments. For block code based redundant index assignments, weak block codes such as a repetition code should be preferred. Simulation results show significant performance improvements.
\end{abstract}

Index Terms-ISCD, index assignment, EXIT chart, SDSD.

\section{INTRODUCTION}

W ITH the discovery of Turbo codes channel decoding close to the Shannon limit has become possible with moderate computational complexity. In the past years the Turbo principle has been extended to other receiver components. One of these extensions is iterative source-channel decoding (ISCD), e.g., [1], [2], [3], which allows to exploit the residual redundancy in source codec parameters such as scale factors or predictor coefficients for speech, audio, and video signals in a Turbo process. This residual redundancy occurs due to imperfect source encoding resulting, e.g., from delay constraints. The a priori knowledge on the residual redundancy, e.g., non-uniform probability distribution or autocorrelation, is utilized for error concealment by a derivative of a soft decision source decoder (SDSD) [4], [5], which exchanges extrinsic reliabilities with a channel decoder.

A key element in the optimization of an ISCD scheme is the index assignment (IA) in the source encoder from quantizer levels to bit patterns [6]. In [7] the concept of redundant IAs is presented, which gives a significant performance improvement and enables a multi-mode ISCD system. More results on redundant IAs can be found in [8], [9]. In this letter we analyze the redundant IAs and optimization criteria in [7], [8], [9], propose a novel optimization concept, and demonstrate by simulation that the new simple redundant IAs outperform the previously known ones. The new redundant IAs resemble a simple repetition code in the given example. Some similarities to repeat-accumulate codes [10] can be observed.

\section{THE ISCD SySTEM MODEL}

\section{A. Conventional ISCD}

In Fig. 1 the baseband model of iterative source-channel decoding is depicted. At time instant $\tau$, a source encoder determines a frame $\underline{u}_{\tau}$ consisting of $K_{S}$ source codec parameters

Manuscript received March 10, 2008. The associate editor coordinating the review of this letter and approving it for publication was Dr. C. Charalambous. The RWTH Aachen University was supported by the European Union under Grant FP6-2002-IST-C 020023-2 FlexCode.

T. Clevorn is with Infineon Technologies, Duisburg, Germany (e-mail: thorsten.clevorn@infineon.com).

L. Schmalen and P. Vary are with the RWTH Aachen University, Germany (e-mail: \{schmalen, vary\}@ind.rwth-aachen.de).

M. Adrat is with FGAN, Wachtberg, Germany (e-mail: adrat@fgan.de).

Digital Object Identifier 10.1109/LCOMM.2008.080374.

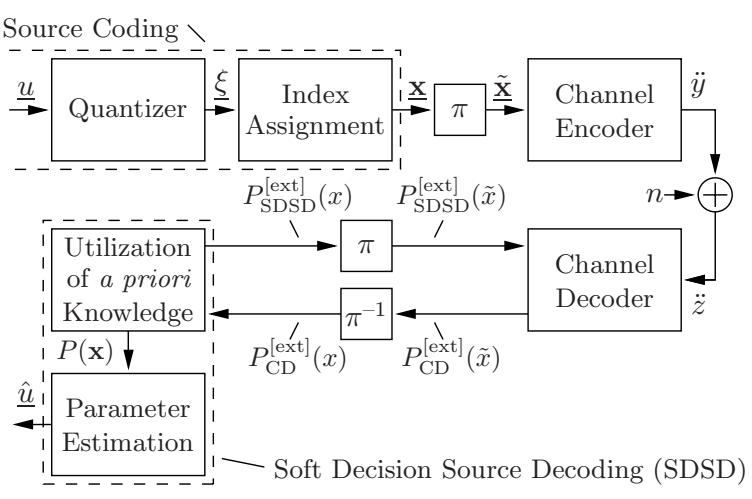

Fig. 1. Baseband model of iterative source-channel decoding

$u_{\kappa, \tau}$ with $\kappa=1, \ldots K_{S}$ denoting the position in the frame. The single elements $u_{\kappa, \tau}$ of $\underline{u}_{\tau}$ are assumed to be statistically independent from each other. By scalar quantization each value $u_{\kappa, \tau}$ is individually mapped to a quantizer reproduction level $\bar{u}_{\kappa}^{\left(\xi_{\kappa}\right)}$ with index $\xi_{\kappa}=0, \ldots Q-1$. The number of quantizer levels is usually assumed as $Q=2^{M}$. To each quantizer level $\bar{u}_{\kappa}^{\left(\xi_{\kappa}\right)}$ selected at time instant $\tau$ a unique bit pattern $\mathbf{x}_{\kappa, \tau}$ of $M$ bits is assigned according to the index assignment $\Gamma$, $\mathbf{x}_{\kappa, \tau}=\Gamma\left(\xi_{\kappa}\right)$. The frame of $K_{S}$ bit patterns is denoted by $\underline{\mathbf{x}}_{\tau}$. The bit interleaver $\pi$ scrambles the incoming frame $\underline{\mathbf{x}}_{\tau}$ of data bits to $\underline{\tilde{x}}_{\tau}$ in a deterministic manner. We restrict the interleaving to a single time frame with index $\tau$ and omit the time frame index $\tau$ in the following where appropriate.

For channel encoding $C$ of $\underline{\tilde{x}}$ we can use, e.g., a standard terminated recursive non-systematic convolutional (RNSC) code of constraint length $J+1$ and rate $r_{\mathrm{C}}$. The resulting codeword is mapped to bipolar bits $\ddot{y} \in\{ \pm 1\}$, e.g., for BPSK transmission with symbol energy $E_{s}=1$. On the channel additive white Gaussian noise (AWGN) $n$ with a known power spectral density of $\sigma_{n}^{2}=N_{0} / 2$ is applied, i.e., $\ddot{z}=\ddot{y}+n$.

The received symbols $\ddot{z}$ are evaluated in a Turbo process, which exchanges extrinsic reliabilities between the channel decoder (CD) and the soft decision source decoder (SDSD). Such reliability information can either be evaluated in terms of probabilities $P(\cdot)$ or as log-likelihood ratios ( $L$-values) $L(\cdot)$.

The ISCD receiver is described in detail, e.g., in [1], [2], [5], [6]. For convolutional codes the equations for the extrinsic probabilities are well known. The SDSD determines extrinsic information mainly from the natural residual source redundancy which remains in the bit patterns $\mathbf{x}_{\kappa, \tau}$ after source encoding. Such residual redundancy appears on parameterlevel, e.g., in terms of a non-uniform distribution $P\left(\mathbf{x}_{\kappa, \tau}\right)$, in terms of correlation, or in any other possible mutual dependency in time $\tau$. The algorithm how to compute the extrinsic $P_{\mathrm{SDSD}}^{[\mathrm{ext}]}(x)$ has been detailed, e.g., in [1], [2], [5], [6].

\section{B. ISCD with Redundant Index Assignments}

Redundant IAs for ISCD were proposed in [7]. In comparison to a conventional ISCD scheme with channel coding of 


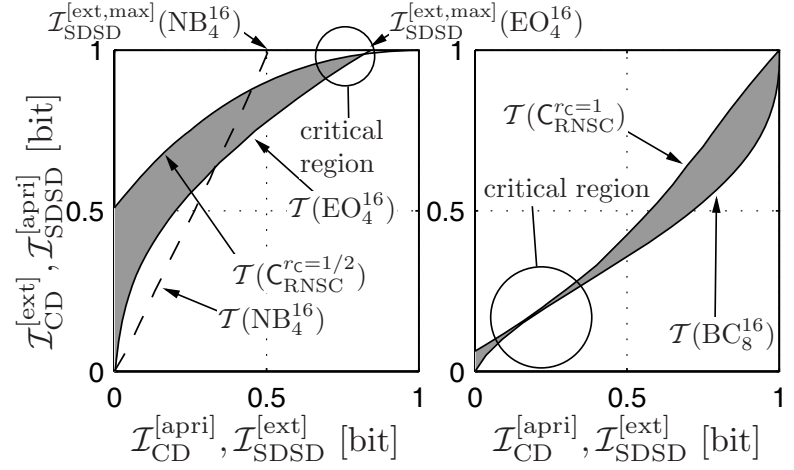

Fig. 2. EXIT chart at $E_{s} / N_{0}=-3.3 \mathrm{~dB}$ for ISCD with conventional IA (left) and redundant IA (right).

rate $r_{\mathrm{C}}$, the number of bits assigned to each quantizer level $\bar{u}_{\kappa}^{\left(\xi_{\kappa}\right)}$ can be increased by a factor of up to $1 / r_{\mathrm{C}}$, assuming a constant gross bit rate by reduction of the channel coding rate at the same time. For example, if $r_{\mathrm{C}}=1 / 2$ channel coding and $M=4$ bits per $Q$ quantizer level were used for conventional ISCD, with redundant IAs and $r_{\mathrm{C}}=1$ bit patterns $\mathbf{x}$ of size $M^{\star}=M / r_{\mathrm{C}}=8$ can be assigned to each quantizer level.

The obvious choice now would be to use $Q^{\star}=2^{M^{\star}}=256$ quantizer levels to reduce the quantization noise. However, the only constraint for $Q^{\star}$ is $Q^{\star} \leq 2^{M^{\star}}$. Thus, if, e.g., the quantization noise of $Q^{\star}=16$ quantization levels is tolerable (as in the conventional case with $M=4$ bits), we can apply a redundant index assignment with $M^{\star}=8$ bits for each level. The benefits of the latter approach with $Q^{\star}<2^{M^{\star}}$ over the obvious choice $Q^{\star}=2^{M^{\star}}$ in case of channel errors have been analyzed in detail in [7]. Such an index assignment with $Q^{\star}<2^{M^{\star}}$ can be considered as a (potentially non-linear) block code with the binary representation of the quantizer level index $\{\xi\}_{2}, \xi=0, \ldots Q^{\star}-1$, as input (see also [11], [12] for noniterative schemes). In case of an index assignment by a linear block code with generator matrix $\mathbf{G}^{\Gamma}$ we get [8]

$$
\mathbf{x}=\Gamma(\xi)=\left(\{\xi\}_{2}\right) \cdot \mathbf{G}^{\Gamma} .
$$

In [8] simple block codes (BC) were chosen with maximal minimum Hamming distance $d_{\min }$. For the exemplary $Q^{\star}=16$ and $M^{\star}=8$ we get for instance

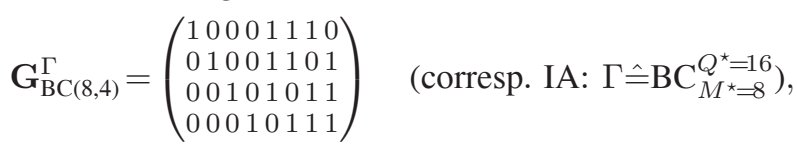

with $d_{\min }\left(\mathbf{G}_{\mathrm{BC}(8,4)}^{\Gamma}\right)=4$. Thus, e.g., for $\xi=3$ we get with (1)

$$
\mathbf{x}=\Gamma(3)=\left(\{3\}_{2}\right) \cdot \mathbf{G}^{\Gamma}=(0011) \cdot \mathbf{G}^{\Gamma}=00111100
$$

\section{OptimizATION OF REDUNDANT INDEX AssignMENTS}

The advantage of redundant IAs with respect to conventional IAs can be best observed in an EXIT chart [6]. In Fig. 2 the EXIT charts of ISCD with a conventional IA and ISCD with a redundant IA are compared. For the simulation settings we refer to Chapter IV. The IAs are identified by $(\cdot)_{M}^{Q}$ or $(\cdot)_{M^{\star}}^{Q^{\star}}$. NB stands for natural binary, EO for EXIT optimized [6], and BC for block coded [8]. In the left EXIT chart we can observe that the EXIT characteristics $\mathcal{T}$ of conventional IAs are limited to a maximum extrinsic mutual information $\mathcal{I}_{\text {SDSD }}^{\text {[ext,max }} \ll 1$ bit [6]. As the stopping intersection with the channel code is usually close to $\mathcal{I}_{\text {SDSD }}^{\text {apri] }}=1$ bit, the maximization of $\mathcal{I}_{\text {SDSD }}^{[\text {ext,max] }}$ is a very good optimization criterion [6].

In contrast, the EXIT characteristics of redundant IAs can reach the point $(1,1)$ in the EXIT chart, if the minimum Hamming distance $d_{\min }$ between the bit patterns $\mathbf{x}$ of the IA is larger than 1 , i.e., $d_{\min } \geq 2$ [13], [8], [14], [9]. Note, for unequally distributed bits $x$ actually only the point $(H(x), H(x))$ can be reached, with $H(x)$ being the entropy of $x$. Both EXIT characteristics $\mathcal{T}\left(\mathrm{C}_{\mathrm{RNSC}}^{r_{\mathrm{C}}}\right)$ of the channel codes reach $(1,1)$, but due to the different $r_{\mathrm{C}}$, they differ significantly.

While in the left plot with the conventional IA the point $(1,1)$ can never be approached by the decoding trajectory at all due to $\mathcal{I}_{\text {SDSD }}^{\text {[ext,max }}<1$, the point $(1,1)$ can be reached in the right plot with the redundant IA as soon as the tunnel opens noticeably, which is the case for a $E_{s} / N_{0}$ slightly above $-3.3 \mathrm{~dB}$. Thus, the critical region is now at a relatively low $\mathcal{I}_{\text {SDSD }}^{\text {[apri] }}$, around $\mathcal{I}_{\text {SDSD }}^{\text {[apri] }} \approx 0.2$ bit, where the tunnel opens, because any IA with $d_{\min } \geq 2$ guarantees reaching $(1,1)$.

The block coded IAs in [8] were chosen for a high $d_{\min }$, e.g., $d_{\min }\left(\mathbf{G}_{\mathrm{BC}(8,4)}^{\Gamma}\right)=4$. The EXIT optimized IAs of [9] (not to be confused with the EXIT optimized IAs of [6]) result actually in a single parity-check code (SPC) for $\mathbf{G}^{\Gamma}$ ("Mapping 2" in [9], cmp. [13], [14] and $\mathbf{G}_{\mathrm{BC}(6,5)}^{\Gamma}$ in [15]). Note, a SPC is the only possibility to achieve $d_{\min } \geq 2$ for $M^{\star}=M+1$ as used in [13], [14], [9], when the IA shall be systematic, i.e., $\{\xi\}_{2}$ is part of $\mathbf{x}$. The proof is obvious. If two $\{\xi\}_{2}$ differ by only one bit of the $M$ bits, one has an even Hamming weight, the other one an odd. If the two corresponding $\mathrm{x}$ with $M^{\star}=M+1$ bit shall have $d_{\min } \geq 2$, the added bit must be different for the two $\mathbf{x}$. Afterwards, both $\mathbf{x}$ have an even weight or both have an odd weight. As this holds for all combinations of $\{\xi\}_{2}$, this results in an even or odd SPC for $\mathbf{G}^{\Gamma}$.

\section{A. Novel Repetition Coded Index Assignments}

If a redundant IA is linear, i.e., (1) can be used, it can be considered as a combination of a conventional natural binary IA, e.g., $\mathrm{NB}_{4}^{16}$ in Fig. 2, and a block code with a generator matrix $\mathbf{G}^{\Gamma}$. Although the EXIT characteristic $\mathcal{T}$ of the final redundant IA cannot be directly computed from the EXIT characteristics $\mathcal{T}\left(\mathrm{NB}_{M}^{Q}\right)$ and $\mathcal{T}\left(\mathbf{G}^{\Gamma}\right)$ of the components, it is worth to take a closer look at $\mathcal{T}\left(\mathbf{G}^{\Gamma}\right)$. In Fig. 3 the $\mathcal{T}\left(\mathbf{G}^{\Gamma}\right)$ of the $\mathrm{BC}(8,4)$ of $(2)$ and of a repetition code,

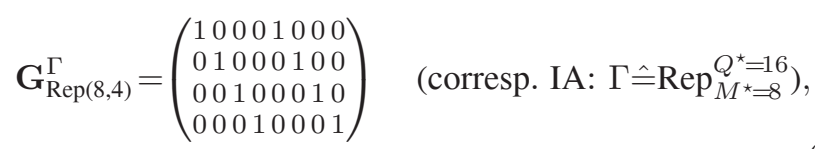

with $d_{\min }\left(\mathbf{G}_{\operatorname{Rep}(8,4)}^{\Gamma}\right)=2$, are depicted in the left subplot. Note the swapped axes. It can be observed that $\mathbf{G}_{\operatorname{Rep}(8,4)}^{\Gamma}$ yields the highest $\mathcal{I}_{\mathbf{G}^{\Gamma}}^{[\mathrm{ext}]}$ for $\mathcal{I}_{\mathbf{G}^{\Gamma}}^{[\text {apri] }}<0.5$ bit and $\mathbf{G}_{\mathrm{BC}^{\Gamma}(8,4)}^{\Gamma}$ the lowest. For $\mathcal{I}_{\mathbf{G}^{\Gamma}}^{\text {aapri] }}>0.5$ bit the order reverses.

A similar behavior can be observed in the right subplot of Fig. 3 for the corresponding redundant IAs, $\mathrm{BC}_{8}^{16}$ and $\operatorname{Rep}_{8}^{16}$. For $\mathcal{I}_{\text {SDSD }}^{\text {[apri] }}>0.5$ bit all redundant IAs provide a sufficient gap to $\mathcal{T}\left(\mathrm{C}_{\mathrm{RNSC}}^{r_{\mathrm{C}}=1}\right)$, as all IAs fulfill $d_{\min } \geq 2$. For $\mathcal{I}_{\mathrm{SDSD}}^{\text {apri] }} \approx 0.2 \mathrm{bit}$ the gap is almost closed for $\mathrm{BC}_{8}^{16}$ at $E_{s} / N_{0}=-3.3 \mathrm{~dB}$, while a sufficient tunnel still exists for $\operatorname{Rep}_{8}^{16}$. This advantage 

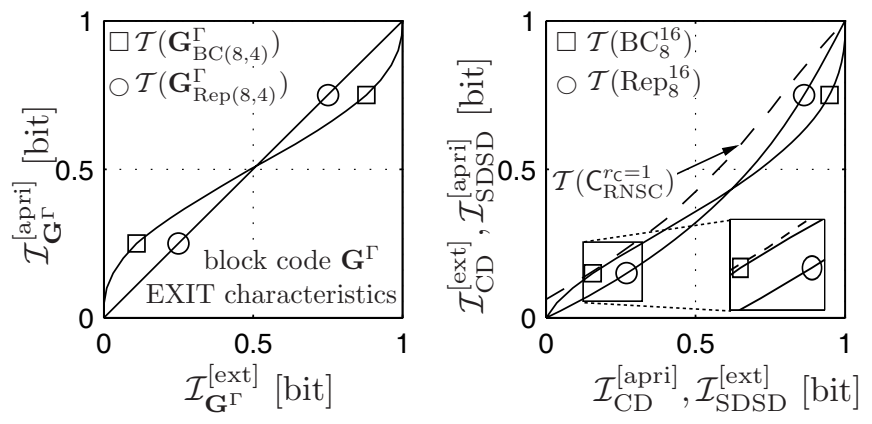

Fig. 3. EXIT characteristics for block codes $\mathbf{G}^{\Gamma}$ (left) and EXIT chart at $E_{s} / N_{0}=-3.3 \mathrm{~dB}$ with corresponding redundant IAs (right).

of $\operatorname{Rep}_{8}^{16}$ can be again found in the simulation results in Chapter IV.

To summarize, for redundant IAs with $d_{\min } \geq 2$ the critical region of the EXIT characteristic is at low $\mathcal{I}_{\text {SDSD }}^{\text {[apri] }}$, i.e., $\mathcal{I}_{\text {SDSD }}^{\text {[apri] }}<0.5$ bit, and not at high $\mathcal{I}_{\text {SDSD }}^{\text {[apri] }}$. The EXIT characteristic of an underlying block code $\mathrm{G}^{\Gamma}$ can serve as indication of the EXIT characteristic of the redundant IA. Block codes $\mathbf{G}^{\Gamma}$ with a low $d_{\text {min }}$ (but $\geq 2$ ), such as a repetition code, provide the best performance in the critical region of the EXIT characteristic, and thus, also the best system performance.

\section{Simulation Results}

The capabilities of the repetition code IAs shall be demonstrated by simulation. Similar settings as in, e.g., [5], [6], [8] are used. $K_{S}=250$ statistically independent parameters per frame $\underline{u}_{\tau}$, modeled by independent Gauss-Markov processes with $\sigma_{u}^{2}=1$ and auto-correlation $\rho=0.9$, are LloydMax quantized to $Q=Q^{\star}=16$ levels with $M=4$ or $M^{\star}=8$ bits/parameter. RNSC codes with the generator polynomials $\mathbf{G}_{\mathrm{C}}^{r_{\mathrm{C}}=1 / 2}=\left(\frac{1+D+D^{3}}{1+D+D^{2}+D^{3}}, \frac{1+D^{2}+D^{3}}{1+D+D^{2}+D^{3}}\right)$ for $r_{\mathrm{C}}=1 / 2$ and $\mathbf{G}_{\mathrm{C}}^{r_{\mathrm{C}}=1}=\left(\frac{1}{1+D+D^{2}+D^{3}}\right)$ for $r_{\mathrm{C}}=1$ are used for channel coding.

In the simulation results in Fig. 4 it can be observed that all ISCD schemes with 25 iterations outperform the best non-iterative scheme by several $\mathrm{dB}$ in $E_{s} / N_{0}$. Note that the depicted non-iterative scheme uses SDSD [4]. Today's systems with hard output channel decoding perform even worse.

The light gray area marks the gain by the known redundant IA $\mathrm{BC}_{8}^{16}$. The maximum parameter $\mathrm{SNR}$ can be retained to much lower $E_{s} / N_{0}$ than with an optimized conventional IA $\mathrm{EO}_{4}^{16}$. The dark gray area marks the additional gain by the new repetition coded IA $\operatorname{Rep}_{8}^{16}$. On the one hand, the gap to the theoretical limit is closed. The limit is the OPTA-SPB bound [16], the optimum performance theoretically attainable using the sphere packing bound to incorporate the finite block size. On the other hand, the performance in the waterfall region is also improved. The gap to ISCD with a conventional IA $\left(\mathrm{EO}_{4}^{16}\right)$ is closed from $\Delta_{E_{s} / N_{0}} \approx 0.8 \mathrm{~dB}$ for $\mathrm{BC}_{8}^{16}$. Thus, the new repetition coded IA $\operatorname{Rep}_{8}^{16}$ outperforms the known IAs, an optimized conventional IA such as $\mathrm{EO}_{4}^{16}$ as well as redundant IAs such as $\mathrm{BC}_{8}^{16}$, in the whole relevant $E_{s} / N_{0}$ range.

\section{CONClusion}

We presented an analysis of redundant IAs for ISCD. Based on EXIT charts new design and optimization criteria are derived. The extrinsic mutual information for low to medium

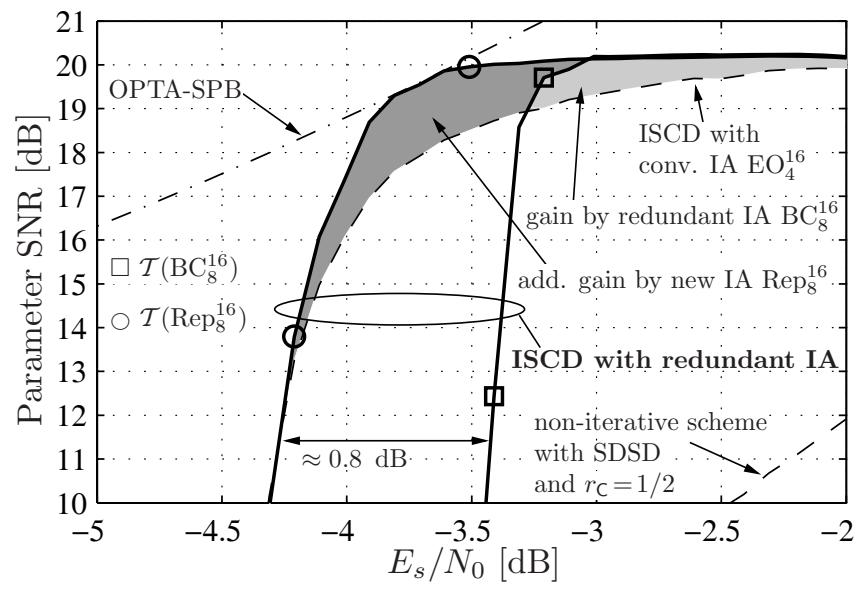

Fig. 4. Parameter SNR simulation results.

a priori information should be maximized. We show that weak block codes such as simple repetition codes fulfill this guideline best. Simulation results show a significant performance improvement with a repetition coded redundant IA compared to known redundant and conventional IAs.

\section{REFERENCES}

[1] M. Adrat, P. Vary, and J. Spittka, "Iterative source-channel decoder using extrinsic information from softbit-source decoding," in Proc. IEEE ICASSP, vol. 4, Salt Lake City, Utah, May 2001, pp. 2653-2656.

[2] N. Görtz, "On the iterative approximation of optimal joint sourcechannel decoding," IEEE J. Select. Areas Commun., vol. 19, pp. 1662 1670, Sept. 2001.

[3] P. Vary and R. Martin, Digital Speech Transmission-Enhancement, Coding \& Error Concealment. Chichester, UK: John Wiley \& Sons, Ltd., 2006.

[4] T. Fingscheidt and P. Vary, "Softbit speech decoding: a new approach to error concealment," IEEE Trans. Speech Audio Processing, vol. 9, pp. 240-251, Mar. 2001.

[5] R. Martin, U. Heute, and C. Antweiler, Advances in Digital Speech Transmission. Chichester, UK: John Wiley \& Sons, Ltd., 2008.

[6] M. Adrat and P. Vary, "Iterative source-channel decoding: improved system design using EXIT charts," EURASIP J. Appl. Signal Processin (Special Issue: Turbo Processing), pp. 928-941, May 2005.

[7] M. Adrat, P. Vary, and T. Clevorn, "Optimized bit rate allocation for iterative source-channel decoding and its extension towards multi-mode transmission," in Proc. 14th IST Mob. \& Wirel. Comm. Summit, Dresden, Germany, June 2005.

[8] T. Clevorn, P. Vary, and M. Adrat, "Iterative source-channel decoding using short block codes," in Proc. IEEE ICASSP, vol. 4, Toulouse, France, May 2006, pp. 221-224.

[9] A. Q. Pham, L. L. Yang, and L. Hanzo, "Joint optimization of iterative source and channel decoding using over-complete source-mapping," in Proc. IEEE VTC-Fall, Baltimore, MD, USA, Sept. 2007, pp. 1072-1076.

[10] D. Divsalar, H. Jin, and R. J. McEliece, "Coding theorems for 'turbolike' codes," in Proc. Allerton Conf. on Communication, Control and Computing, Monticello, IL, USA, Sept. 1998.

[11] T. Fingscheidt, S. Heinen, and P. Vary, "Joint speech codec parameter and channel decoding of parameter individual block codes (PIBC)," in Proc. IEEE SCW, Porvoo, Finland, June 1999, pp. 75-77.

[12] S. Heinen and P. Vary, "Source-optimized channel coding for digital transmission channels," IEEE Trans. Commun., vol. 53, pp. 592-600, May 2005.

[13] J. Kliewer, N. Görtz, and A. Mertins, "Iterative source-channel decoding with Markov random field source models," IEEE Trans. Signal Processing, vol. 54, pp. 3688-3701, Oct. 2006.

[14] R. Thobaben, "A new transmitter concept for iteratively-decoded sourcechannel coding schemes," in Proc. IEEE SPAWC 2007, Helsinki, Finland, June 2007, pp. 1-5.

[15] T. Clevorn, M. Adrat, and P. Vary, "Turbo decodulation using highly redundant index assignments and multi-dimensional mappings," in Proc. 4th Inter. Symp. on Turbo Codes, Munich, Germany, Apr. 2006.

[16] T. Clevorn, L. Schmalen, P. Vary, and M. Adrat, "On the optimum performance theoretically attainable for scalarly quantized correlated sources," in Proc. ISITA, Seoul, Korea, Oct. 2006. 